\title{
Complex interpolation of variable Triebel-Lizorkin spaces
}

\author{
Douadi Drihem \\ Department of Mathematics, \\ Laboratory of Functional Analysis and Geometry of spaces, \\ M'sila University, M'sila, Algeria \\ douadidr@yahoo.fr
}

September 15, 2021

\begin{abstract}
We study complex interpolation of variable Triebel-Lizorkin spaces, especially we present the complex interpolation of $F_{p(\cdot), q}^{\alpha}$ and $F_{p(\cdot), p(\cdot)}^{\alpha(\cdot)}$ spaces. Also, some limiting cases are given.

MSC classification: 46E35, 26B35.

Key words and phrases: Triebel-Lizorkin spaces, Complex interpolation, Calderón products, Variable exponents.
\end{abstract}

\section{Introduction}

Interpolation of spaces have been a central topic in analysis, and are now of increasing applications in many fields of mathematics especially harmonic analysis and partial differential equations. For more details on this topic we refer the reader to Bergh and Löfström [2], and Triebel [17], where the complex interpolation for Besov and/or Triebel-Lizorkin spaces are given. The main purpose of this paper is to establish the complex interpolation for variable Triebel-Lizorkin spaces. Firstly we use the so-called retraction method to present interpolation results in variable Triebel-Lizorkin spaces $F_{p(\cdot), q}^{\alpha}$. Secondly we shall apply a method which has been used by [10] and [16], where we shall calculate the Calderón products of associated sequence spaces. Then, from an abstract theory on the relation between the complex interpolation and the Calderón product of Banach lattices obtained by Calderón [3], Frazier, Jawerth [10], Mendez, Mitrea [13] and Kalton, Maybororda, Mitrea [11], we deduce the complex interpolation theorems of these sequence spaces. Under some assumptions the complex interpolation theorems for $F_{p(\cdot), p(\cdot)}^{\alpha(\cdot)}$ are lifted by the $\varphi$-transforms characterization of variable TriebelLizorkin spaces. Finally we will present and briefly discuss some results concerning the complex interpolation for the spaces $F_{p(\cdot), q(\cdot)}^{\alpha(\cdot)}$.

As usual, we denote by $\mathbb{R}^{n}$ the $n$-dimensional real Euclidean space, $\mathbb{N}$ the collection of all natural numbers and $\mathbb{N}_{0}:=\mathbb{N} \cup\{0\}$. The letter $\mathbb{Z}$ stands for the set of all integer 
numbers. The expression $f \lesssim g$ means that $f \leq c g$ for some independent constant $c$ (and non-negative functions $f$ and $g$ ), and $f \approx g$ means $f \lesssim g \lesssim f$.

By supp $f$ we denote the support of the function $f$, i.e., the closure of its non-zero set. If $E \subset \mathbb{R}^{n}$ is a measurable set, then $|E|$ stands for the (Lebesgue) measure of $E$ and $\chi_{E}$ denotes its characteristic function.

For $v \in \mathbb{Z}$ and $m=\left(m_{1}, \ldots, m_{n}\right) \in \mathbb{Z}^{n}$, let $Q_{v, m}$ be the dyadic cube in $\mathbb{R}^{n}, Q_{v, m}:=$ $\left\{\left(x_{1}, \ldots, x_{n}\right): m_{i} \leq 2^{v} x_{i}<m_{i}+1, i=1,2, \ldots, n\right\}$. For the collection of all such cubes we use $\mathcal{Q}:=\left\{Q_{v, m}: v \in \mathbb{Z}, m \in \mathbb{Z}^{n}\right\}$. For each cube $Q$, we denote by $x_{v, m}$ the lower left-corner $2^{-v} m$ of $Q=Q_{v, m}$ and its side length by $l(Q)$. Furthermore, we put $v_{Q}:=-\log _{2} l(Q), v_{Q}^{+}:=\max \left(v_{Q}, 0\right)$ and $\chi_{Q_{v, m}}=\chi_{v, m}, v \in \mathbb{Z}, m \in \mathbb{Z}^{n}$.

The symbol $\mathcal{S}\left(\mathbb{R}^{n}\right)$ is used in place of the set of all Schwartz functions on $\mathbb{R}^{n}$. We define the Fourier transform of a function $f \in \mathcal{S}\left(\mathbb{R}^{n}\right)$ by

$$
\mathcal{F}(f)(\xi):=(2 \pi)^{-n / 2} \int_{\mathbb{R}^{n}} e^{-i x \cdot \xi} f(x) d x, \quad \xi \in \mathbb{R}^{n} .
$$

We denote by $\mathcal{S}^{\prime}\left(\mathbb{R}^{n}\right)$ the dual space of all tempered distributions on $\mathbb{R}^{n}$. For $v \in \mathbb{Z}$, $\varphi \in \mathcal{S}\left(\mathbb{R}^{n}\right)$ and $x \in \mathbb{R}^{n}$, we set $\widetilde{\varphi}(x):=\overline{\varphi(-x)}, \varphi_{v}(x):=2^{v n} \varphi\left(2^{v} x\right)$, and

$$
\varphi_{v, m}(x):=2^{v n / 2} \varphi\left(2^{v} x-m\right)=\left|Q_{v, m}\right|^{1 / 2} \varphi_{v}\left(x-x_{v, m}\right) \quad \text { if } \quad Q=Q_{v, m} .
$$

The variable exponents that we consider are always measurable functions $p$ on $\mathbb{R}^{n}$ with range in $\left[c, \infty\right.$ [ for some $c>0$. We denote the set of such functions by $\mathcal{P}_{0}$. The subset of variable exponents with range $[1, \infty[$ is denoted by $\mathcal{P}$. We use the standard notation $p^{-}:=\underset{x \in \mathbb{R}^{n}}{\operatorname{ess-inf}} p(x)$ and $p^{+}:=\underset{x \in \mathbb{R}^{n}}{\operatorname{ess-sup}} p(x)$.

The variable exponent modular is defined by $\varrho_{p(\cdot)}(f):=\int_{\mathbb{R}^{n}} \rho_{p(x)}(|f(x)|) d x$, where $\rho_{p}(t)=t^{p}$. The variable exponent Lebesgue space $L^{p(\cdot)}$ consists of measurable functions $f$ on $\mathbb{R}^{n}$ such that $\varrho_{p(\cdot)}(\lambda f)<\infty$ for some $\lambda>0$. We define the Luxemburg (quasi)norm on this space by the formula $\|f\|_{p(\cdot)}:=\inf \left\{\lambda>0: \varrho_{p(\cdot)}\left(\frac{f}{\lambda}\right) \leq 1\right\}$. A useful property is that $\|f\|_{p(\cdot)} \leq 1$ if and only if $\varrho_{p(\cdot)}(f) \leq 1$, see [9], Lemma 3.2.4.

Let $p, q \in \mathcal{P}_{0}$. The Lebesgue-sequence space $L^{p(\cdot)}\left(\ell_{q(\cdot)}\right)$ is defined to be the space of all family of functions $f_{v}, v \geq 0$ such that

$$
\left\|\left(f_{v}\right)_{v \geq 0}\right\|_{L^{p(\cdot)}\left(\ell_{q(\cdot)}\right)}:=\|\|\left(f_{v}(x)\right)_{v \geq 0}\left\|_{\ell_{q(x)}}\right\|_{p(\cdot)} .
$$

It is easy to show that $L^{p(\cdot)}\left(\ell_{q(\cdot)}\right)$ is always a quasi-normed space and it is a normed space, if $\min (p(x), q(x)) \geq 1$ holds point-wise.

We say that $g: \mathbb{R}^{n} \rightarrow \mathbb{R}$ is locally $\log$-Hölder continuous, abbreviated $g \in C_{\text {loc }}^{\text {log }}$, if there exists $c_{\log }(g)>0$ such that

$$
|g(x)-g(y)| \leq \frac{c_{\log }(g)}{\log (e+1 /|x-y|)}
$$

for all $x, y \in \mathbb{R}^{n}$. We say that $g$ satisfies the log-Hölder decay condition, if there exists $g_{\infty} \in \mathbb{R}$ and a constant $c_{\log }>0$ such that

$$
\left|g(x)-g_{\infty}\right| \leq \frac{c_{\log }}{\log (e+|x|)}
$$




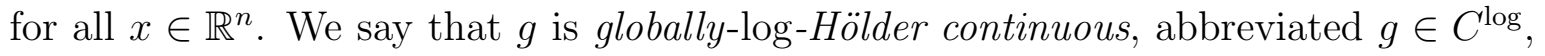
if it is locally log-Hölder continuous and satisfies the log-Hölder decay condition. The constants $c_{\log }(g)$ and $c_{\log }$ are called the locally log-Hölder constant and the log-Hölder decay constant, respectively. We note that all functions $g \in C_{\mathrm{loc}}^{\log }$ always belong to $L^{\infty}$. It is known that for $p \in C^{\log }$ we have

$$
\left\|\chi_{B}\right\|_{p(\cdot)}\left\|\chi_{B}\right\|_{p^{\prime}(\cdot)} \approx|B|
$$

Also,

$$
\left\|\chi_{B}\right\|_{p(\cdot)} \approx|B|^{\frac{1}{p(x)}}, \quad x \in B
$$

for small balls $B \subset \mathbb{R}^{n}\left(|B| \leq 2^{n}\right)$, with constants only depending on the log-Hölder constant of $p$ (see, for example, [9, Section 4.5]). Here $p^{\prime}$ denotes the conjugate exponent of $p$ given by $1 / p(\cdot)+1 / p^{\prime}(\cdot)=1$.

Recall that $\eta_{v, m}(x):=2^{n v}\left(1+2^{v}|x|\right)^{-m}$, for any $x \in \mathbb{R}^{n}, v \in \mathbb{N}_{0}$ and $m>0$. Note that $\eta_{v, m} \in L^{1}$ when $m>n$ and that $\left\|\eta_{v, m}\right\|_{1}=c_{m}$ is independent of $v$.

\subsection{Basic tools}

In this subsection we present some results which are useful for us. The following lemma is from [8, Lemma 6.1], see also [14, Lemma 19].

Lemma 1 Let $\alpha \in C_{\mathrm{loc}}^{\log }$ and let $R \geq c_{\log }(\alpha)$, where $c_{\log }(\alpha)$ is the constant from (1) for $\alpha$. Then

$$
2^{v \alpha(x)} \eta_{v, h+R}(x-y) \leq c 2^{v \alpha(y)} \eta_{v, h}(x-y)
$$

with $c>0$ independent of $x, y \in \mathbb{R}^{n}$ and $v, h \in \mathbb{N}_{0}$.

The previous lemma allows us to treat the variable smoothness in many cases as if it were not variable at all, namely we can move the term inside the convolution as follows:

$$
2^{v \alpha(x)} \eta_{v, h+R} *|f|(x) \leq c \eta_{v, h} *\left(2^{v \alpha(\cdot)}|f|\right)(x) .
$$

Lemma 2 Let $p, q \in C^{\log }$ with $1<p^{-} \leq p^{+}<\infty$ and $1<q^{-} \leq q^{+}<\infty$. For $m>n$, there exists $c>0$ such that

$$
\left\|\left(\eta_{v, m} * f_{v}\right)_{v}\right\|_{L^{p(\cdot)}\left(\ell_{q(\cdot)}\right)} \leq c\left\|\left(f_{v}\right)_{v}\right\|_{L^{p(\cdot)}\left(\ell_{q(\cdot)}\right)} .
$$

The proof is given in [8, Theorem 3.2].

Now we introduce the following sequence space.

Definition 1 Let $p, q \in \mathcal{P}_{0}$ where $0<p^{+}, q^{+}<\infty$ and let $\alpha: \mathbb{R}^{n} \rightarrow \mathbb{R}$. Then for all complex valued sequences $\lambda:=\left\{\lambda_{v, m}\right\}_{v \in \mathbb{N}_{0}, m \in \mathbb{Z}^{n}} \subset \mathbb{C}$ we define

$$
f_{p(\cdot), q(\cdot)}^{\alpha(\cdot)}:=\left\{\lambda:\|\lambda\|_{f_{p(\cdot), q(\cdot)}^{\alpha()}}<\infty\right\}
$$

where

$$
\|\lambda\|_{f_{p(\cdot), q(\cdot)}^{\alpha(\cdot)}}:=\left\|\left(\sum_{m \in \mathbb{Z}^{n}} 2^{v\left(\alpha(\cdot)+\frac{n}{2}\right)} \lambda_{v, m} \chi_{v, m}\right)_{v \geq 0}\right\|_{L^{p(\cdot)}\left(\ell_{q(\cdot)}\right)}
$$


and

$$
f_{\infty, q}^{\alpha(\cdot)}:=\left\{\lambda:\|\lambda\|_{f_{\infty, q}^{\alpha(\cdot)}}<\infty\right\}
$$

where

$$
\|\lambda\|_{f_{\infty, q}^{\alpha(\cdot)}}:=\sup _{Q \in \mathcal{Q}} \frac{1}{|Q|^{1 / q}}\left(\sum_{v=v_{Q}^{+}}^{\infty} \int_{Q} \sum_{m \in \mathbb{Z}^{n}} 2^{v\left(\alpha(x)+\frac{n}{2}\right) q}\left|\lambda_{v, m}\right|^{q} \chi_{v, m}(x) d x\right)^{1 / q}
$$

Notice that the supremum can be taken respect to dyadic cubes with side length $\leq 1$.

Lemma 3 Let $\alpha \in C_{\operatorname{loc}}^{\log }, p, q \in C^{\log }, 0<p^{+}, q^{+}<\infty, j \in \mathbb{N}_{0}, m \in \mathbb{Z}^{n}$ and $x \in Q_{j, m}$. Let $\lambda \in f_{p(\cdot), q(\cdot)}^{\alpha(\cdot)}$. Then there exists $c>0$ independent of $j$ and $m$ such that

$$
\left|\lambda_{j, m}\right| \leq c 2^{-j\left(\alpha(x)-\frac{n}{p(x)}+\frac{n}{2}\right)}\|\lambda\|_{f_{p(\cdot), q(\cdot)}^{\alpha(\cdot)}}
$$

Proof. Let $\lambda \in f_{p(\cdot), q(\cdot)}^{\alpha(\cdot)}, j \in \mathbb{N}_{0}, m \in \mathbb{Z}^{n}$ and $x \in Q_{j, m}$. Using the fact that $2^{j(\alpha(x)-\alpha(y))} \leq c$ for any $x, y \in Q_{j, m}$, we obtain

$$
\begin{aligned}
2^{j \alpha(x) p^{-}}\left|\lambda_{j, m}\right|^{p^{-}} & =\left|Q_{j, m}\right|^{-1} \int_{Q_{j, m}} 2^{j \alpha(x) p^{-}}\left|\lambda_{j, m}\right|^{p^{-}} \chi_{j, m}(y) d y \\
& \leq c\left|Q_{j, m}\right|^{-1} \int_{Q_{j, m}} 2^{j \alpha(y) p^{-}}\left|\lambda_{j, m}\right|^{p^{-}} \chi_{j, m}(y) d y .
\end{aligned}
$$

Applying Hölder's inequality to estimate this expression by

$$
c\left|Q_{j, m}\right|^{-1}\left\|2^{j \alpha(\cdot) p^{-}}\left|\lambda_{j, m}\right|^{p^{-}} \chi_{j, m}\right\|_{p / p^{-}}\left\|\chi_{j, m}\right\|_{\left(p / p^{-}\right)^{\prime}} \leq c\|\lambda\|_{f_{p(\cdot), q(\cdot)}^{\alpha(\cdot)}}^{p^{-}}\left\|\chi_{j, m}\right\|_{p / p^{-}}^{-1} 2^{-j \frac{n p^{-}}{2}}
$$

where we have used (2). Therefore for any $x \in Q_{j, m}$

$$
\left|\lambda_{j, m}\right| \leq c 2^{-j\left(\alpha(x)-\frac{n}{p(x)}+\frac{n}{2}\right)}\|\lambda\|_{f_{p(\cdot), q(\cdot)}^{\alpha(\cdot)}},
$$

by (3), which completes the proof.

Proposition 1 Let $\alpha \in C_{\text {loc }}^{\log }$. Then $\lambda=\left\{\lambda_{v, m} \in \mathbb{C}\right\}_{v \in \mathbb{N}_{0}, m \in \mathbb{Z}^{n}} \in f_{\infty, q}^{\alpha(\cdot)}$ if and only if for each dyadic cube $Q_{v, m}$ there is a subset $E_{Q_{v, m}} \subset Q_{v, m}$ with $\left|E_{Q_{v, m}}\right|>\left|Q_{v, m}\right| / 2$ (or any other, fixed, number $0<\varepsilon<1$ ) such that

$$
\left\|\left(\sum_{v=0}^{\infty} \sum_{m \in \mathbb{Z}^{n}} 2^{v\left(\alpha(\cdot)+\frac{n}{2}\right) q}\left|\lambda_{v, m}\right|^{q} \chi_{E_{v, m}}\right)^{1 / q}\right\|_{\infty}<\infty .
$$

Moreover, the infimum of this expression over all such collections $\left\{E_{Q_{v, m}}\right\}_{v, m}$ is equivalent to $\|\lambda\|_{f_{\infty, q}^{\alpha(\cdot)}}$.

The proof is given in [6]. We will make use of the following statement, see [7], Lemma 3.3 . 
Lemma 4 Let $p \in C^{\log }$ with $1 \leq p^{-} \leq p^{+}<\infty$. Then for every $m>0$ there exists $\gamma=e^{-2 m / c_{\log }(1 / p)} \in(0,1)$ such that

$$
\left(\frac{\gamma}{|Q|} \int_{Q}|f(y)| d y\right)^{p(x)} \leq \frac{1}{|Q|} \int_{Q}|f(y)|^{p(y)} d y+\min \left(|Q|^{m}, 1\right) g(x)
$$

for every cube (or ball) $Q \subset \mathbb{R}^{n}$, all $x \in Q \subset \mathbb{R}^{n}$ and all $f \in L^{p(\cdot)}+L^{\infty}$ with $\|f\|_{p(\cdot)}+$ $\|f\|_{\infty} \leq 1$, where

$$
g(x):=(e+|x|)^{-m}+\frac{1}{|Q|} \int_{Q}(e+|y|)^{-m} d y .
$$

Notice that in the proof of this theorem we need only that

$$
\int_{Q}|f(y)|^{p(y)} d y \leq 1
$$

and/or $\|f\|_{\infty} \leq 1$. Moreover if $|Q| \leq 1$, we have $g(x) \leq c \eta_{0, m}(x)$ for any $x \in Q$, where $c>0$ is independent of $x$ and $m$.

\section{Variable Triebel-Lizorkin spaces}

The definition of Triebel-Lizorkin spaces of variable smoothness and integrability is based on the technique of decomposition of unity exactly in the same manner as in the case of constant exponents. Select a pair of Schwartz functions $\Phi$ and $\varphi$ satisfy

$$
\operatorname{supp} \mathcal{F} \Phi \subset \overline{B(0,2)} \text { and }|\mathcal{F} \Phi(\xi)| \geq c \text { if }|\xi| \leq \frac{5}{3}
$$

and

$$
\operatorname{supp} \mathcal{F} \varphi \subset \overline{B(0,2)} \backslash B(0,1 / 2) \text { and }|\mathcal{F} \varphi(\xi)| \geq c \text { if } \frac{3}{5} \leq|\xi| \leq \frac{5}{3}
$$

where $c>0$. It easy to see that $\int_{\mathbb{R}^{n}} x^{\gamma} \varphi(x) d x=0$ for all multi-indices $\gamma \in \mathbb{N}_{0}^{n}$. Now, we define the spaces under consideration.

Definition 2 Let $\alpha: \mathbb{R}^{n} \rightarrow \mathbb{R}$ and $p, q \in \mathcal{P}_{0}$ with $0<p^{+}, q^{+}<\infty$. Let $\Phi$ and $\varphi$ satisfy (5) and (6)), respectively and we put $\varphi_{v}=2^{v n} \varphi\left(2^{v} \cdot\right)$. The Triebel-Lizorkin space $F_{p(\cdot), q(\cdot)}^{\alpha(\cdot)}$ is the collection of all $f \in \mathcal{S}^{\prime}\left(\mathbb{R}^{n}\right)$ such that

$$
\|f\|_{F_{p(\cdot), q(\cdot)}^{\alpha(\cdot)}}:=\left\|\left(2^{v \alpha(\cdot)} \varphi_{v} * f\right)_{v \geq 0}\right\|_{L^{p(\cdot)}\left(\ell_{q(\cdot)}\right)}<\infty
$$

where $\varphi_{0}$ is replaced by $\Phi$.

The Triebel-Lizorkin spaces with variable smoothness have first been introduced in [8] under much more restrictive conditions on $\alpha(\cdot)$. If $p(\cdot), q(\cdot)$ and $\alpha(\cdot)$ are constants, then we derive the well known Triebel-Lizorkin spaces. Taking $\alpha \in \mathbb{R}$ and $q \in(0, \infty]$ as constants we derive the spaces $F_{p(\cdot), q}^{\alpha}$ studied by $\mathrm{Xu}$ in [23] and [24]. The spaces $F_{p(\cdot), q(\cdot)}^{\alpha(\cdot)}$ are independent of the particular choice of the system $\left\{\varphi_{v}\right\}_{v}$ appearing in their definitions. Moreover, if $\alpha \in C_{\mathrm{loc}}^{\log }$ and $p, q \in \mathcal{P}_{0}^{\log }$ with $0<p^{-} \leq p^{+}<\infty$ and $0<q^{-} \leq q^{+}<\infty$, then

$$
\mathcal{S}\left(\mathbb{R}^{n}\right) \hookrightarrow F_{p(\cdot), q(\cdot)}^{\alpha(\cdot)} \hookrightarrow \mathcal{S}^{\prime}\left(\mathbb{R}^{n}\right)
$$


Definition 3 Let $\alpha: \mathbb{R}^{n} \rightarrow \mathbb{R}$ and $0<q<\infty$. Let $\Phi$ and $\varphi$ satisfy (5) and (6) , respectively and we put $\varphi_{v}=2^{v n} \varphi\left(2^{v} \cdot\right)$. The Triebel-Lizorkin space $F_{\infty, q}^{\alpha(\cdot)}$ is the collection of all $f \in \mathcal{S}^{\prime}\left(\mathbb{R}^{n}\right)$ such that

$$
\|f\|_{F_{\infty, q}^{\alpha(\cdot)}}:=\sup _{Q \in \mathcal{Q}} \frac{1}{|Q|^{1 / q}}\left(\sum_{v=v_{Q}^{+}}^{\infty} \int_{Q} 2^{v \alpha(x) q}\left|\varphi_{v} * f(x)\right|^{q} d x\right)^{1 / q}<\infty
$$

where $\varphi_{0}$ is replaced by $\Phi$.

For more information about these function spaces, consult [5] and [6], with different notation. Notice that the supremum can be taken respect to dyadic cubes with side length $\leq 1$. One of the key tools to prove the interpolation property of the spaces is their $\varphi$-transforms characterization, which transfers the problem from function spaces to their corresponding sequence spaces. Let $\Phi$ and $\varphi$ satisfy, respectively (5) and (6) . By [10, pp. 130-131], there exist functions $\Psi \in \mathcal{S}\left(\mathbb{R}^{n}\right)$ satisfying (5) and $\psi \in \mathcal{S}\left(\mathbb{R}^{n}\right)$ satisfying (6) such that for all $\xi \in \mathbb{R}^{n}$

$$
\mathcal{F} \widetilde{\Phi}(\xi) \mathcal{F} \Psi(\xi)+\sum_{j=1}^{\infty} \mathcal{F} \widetilde{\varphi}\left(2^{-j} \xi\right) \mathcal{F} \psi\left(2^{-j} \xi\right)=1, \quad \xi \in \mathbb{R}^{n}
$$

Furthermore, we have the following identity for all $f \in \mathcal{S}^{\prime}\left(\mathbb{R}^{n}\right)$; see [10, (12.4)]

$$
\begin{aligned}
f & =\Psi * \widetilde{\Phi} * f+\sum_{v=1}^{\infty} \psi_{v} * \widetilde{\varphi}_{v} * f \\
& =\sum_{m \in \mathbb{Z}^{n}} \widetilde{\Phi} * f(m) \Psi(\cdot-m)+\sum_{v=1}^{\infty} 2^{-v n} \sum_{m \in \mathbb{Z}^{n}} \widetilde{\varphi}_{v} * f\left(2^{-v} m\right) \psi_{v}\left(\cdot-2^{-v} m\right) .
\end{aligned}
$$

Recall that the $\varphi$-transform $S_{\varphi}$ is defined by setting $\left(S_{\varphi}\right)_{0, m}=\left\langle f, \Phi_{m}\right\rangle$ where $\Phi_{m}(x)=$ $\Phi(x-m)$ and $\left(S_{\varphi}\right)_{v, m}=\left\langle f, \varphi_{v, m}\right\rangle$ where $\varphi_{v, m}(x)=2^{v n / 2} \varphi\left(2^{v} x-m\right)$ and $v \in \mathbb{N}$. The inverse $\varphi$-transform $T_{\psi}$ is defined by

$$
T_{\psi} \lambda:=\sum_{m \in \mathbb{Z}^{n}} \lambda_{0, m} \Psi_{m}+\sum_{v=1}^{\infty} \sum_{m \in \mathbb{Z}^{n}} \lambda_{v, m} \psi_{v, m}
$$

where $\lambda:=\left\{\lambda_{v, m}\right\}_{v \in \mathbb{N}_{0}, m \in \mathbb{Z}^{n}} \subset \mathbb{C}$, see [10].

Now we present the $\varphi$-transform characterization of these function spaces, see [8] and 22 .

Theorem 1 Let $\alpha \in C_{\mathrm{loc}}^{\log }$ and $p, q \in C^{\log }$ with $0<p^{+}, q^{+}<\infty$. Suppose that $\Phi$, $\Psi \in \mathcal{S}\left(\mathbb{R}^{n}\right)$ satisfy (5) and $\varphi, \psi \in \mathcal{S}\left(\mathbb{R}^{n}\right)$ satisfy (6) such that (9) holds. The operators $S_{\varphi}: F_{p(\cdot), q(\cdot)}^{\alpha(\cdot)} \rightarrow f_{p(\cdot), q(\cdot)}^{\alpha(\cdot)}$ and $T_{\psi}: f_{p(\cdot), q(\cdot)}^{\alpha(\cdot)} \rightarrow F_{p(\cdot), q(\cdot)}^{\alpha(\cdot)}$ are bounded. Furthermore, $T_{\psi} \circ S_{\varphi}$ is the identity on $F_{p(\cdot), q(\cdot)}^{\alpha(\cdot)}$.

Notice that this theorem is true for $F_{\infty, q}^{\alpha(\cdot)}$ spaces, with $\alpha \in C_{\mathrm{loc}}^{\log }$ and $0<q<\infty$, see [5]. 


\section{Complex interpolation}

In this section we study complex interpolation of variable Triebel-Lizorkin spaces.

\subsection{Complex interpolation for the spaces $F_{p(\cdot), q}^{\alpha}$}

In this subsection we study the complex interpolation of variable Triebel-Lizorkin spaces $F_{p(\cdot), q}^{\alpha}$. We use the so-called retraction method which allows us to reduce the problem to the interpolation of appropriate sequence spaces, see for instance the monographs $[2,17-18]$. More information about complex interpolation of Besov and TriebelLizorkin spaces of fixed exponents can be found in [17], [18], [19] and [20]. See [15] for the complex interpolation (introduced by Triebel [17]) of Besov spaces and TriebelLizorkin spaces with variable exponents. See also [1] for the complex interpolation of variable Besov spaces. Complex interpolation between variable Lebesgue spaces and $B M O$ (or Hardy spaces) is given in [12].

Let $A_{0}:=\{z \in \mathbb{C}: 0<\operatorname{Re} z<1\}$ and $A:=\{z \in \mathbb{C}: 0 \leq \operatorname{Re} z \leq 1\}$.

Definition 4 Let $\left(X_{0}, X_{1}\right)$ be an interpolation couple of Banach lattices. Define $\mathcal{F}\left(X_{0}, X_{1}\right)$ as the space of bounded analytic functions $g: A_{0} \rightarrow X_{0}+X_{1}$, which extend continuously to the closure $A$, such that the functions $t \rightarrow g(j+i t)$ are bounded continuous functions into $X_{j}, j=0,1$, which tend to zero as $|t| \rightarrow \infty$. We endow $\mathcal{F}\left(X_{0}, X_{1}\right)$ with the norm

$$
\|g\|_{\mathcal{F}\left(X_{0}, X_{1}\right)}:=\max \left(\sup _{t}\|g(i t)\|_{X_{0}}, \sup _{t}\|g(1+i t)\|_{X_{1}}\right) \text {. }
$$

Further, we define the complex interpolation space

$$
\left[X_{0}, X_{1}\right]_{\theta}:=\left\{f \in X_{0}+X_{1}: f=g(\theta) \text { for some } g \in \mathcal{F}\left(X_{0}, X_{1}\right)\right\}, \quad 0<\theta<1
$$

and

$$
\|f\|_{\left[X_{0}, X_{1}\right]_{\theta}}:=\inf \left\{\|g\|_{\mathcal{F}\left(X_{0}, X_{1}\right)}: g \in \mathcal{F}\left(X_{0}, X_{1}\right), \quad g(\theta)=f\right\} .
$$

Let $X$ be a complex Banach space. A function $f: \mathbb{R}^{n} \rightarrow X$ is said to be a simple function if it can be written as

$$
f=\sum_{j=1}^{N} a_{j} \chi_{A_{j}}
$$

with $a_{j} \in X$ and pairwise disjoint $A_{j} \subset \mathbb{R}^{n},\left|A_{j}\right|<\infty(j=1, \ldots, N)$. Let $p \in \mathcal{P}$. The Bochner-Lebesgue spaces with variable exponent $L^{p(\cdot)}\left(\mathbb{R}^{n}, X\right)$ is the collection of all measurable functions $f: \mathbb{R}^{n} \rightarrow X$ endowed with the norm:

$$
\|f\|_{L^{p(\cdot)\left(\mathbb{R}^{n}, X\right)}}:=\inf \left\{\lambda>0: \varrho_{L^{p(\cdot)}\left(\mathbb{R}^{n}, X\right)}\left(\frac{f}{\lambda}\right) \leq 1\right\} .
$$

The spaces $L^{p(\cdot)}\left(\mathbb{R}^{n}, X\right)$ have been introduced by C. Cheng and J. Xu [4]. Let $X_{0}$ and $X_{1}$, be two complex Banach spaces, both linearly and continuously embedded in a linear complex Hausdorff space $\mathcal{A}$. Two such Banach spaces are said to be an interpolation couple $\left(X_{0}, X_{1}\right)$. 
Theorem 2 Let $0<\theta<1$. Let $p_{0}, p_{1} \in \mathcal{P}$ with $1 \leq p_{0}^{+}, p_{1}^{+}<\infty$. We put

$$
\frac{1}{p(\cdot)}:=\frac{1-\theta}{p_{0}(\cdot)}+\frac{\theta}{p_{1}(\cdot)}
$$

Further let $\left(X_{0}, X_{1}\right)$ be an interpolation couple. Then

$$
\left[L^{p_{0}(\cdot)}\left(\mathbb{R}^{n}, X_{0}\right), L^{p_{1}(\cdot)}\left(\mathbb{R}^{n}, X_{1}\right)\right]_{\theta}=L^{p(\cdot)}\left(\mathbb{R}^{n},\left[X_{0}, X_{1}\right]_{\theta}\right) .
$$

Proof. Our approach follows essentially [2] and [18]. It is not hard to see, that the space of simple functions is dense in $L^{p_{0}(\cdot)}\left(\mathbb{R}^{n}, X_{0}\right) \cap L^{p_{1}(\cdot)}\left(\mathbb{R}^{n}, X_{1}\right)$, and thus also in $\left.L^{p_{0}(\cdot)}\left(\mathbb{R}^{n}, X_{0}\right), L^{p_{1}(\cdot)}\left(\mathbb{R}^{n}, X_{1}\right)\right]_{\theta}$ by [2, Theorem 4.2.2]. From now we consider only simple functions. Let $f(x) \neq 0$ be a simple function,

$$
f=\sum_{j=1}^{N} a_{j} \chi_{A_{j}}
$$

with $a_{j} \in X_{0} \cap X_{1}$ and pairwise disjoint $A_{j} \subset \mathbb{R}^{n},\left|A_{j}\right|<\infty(j=1, \ldots, N)$. Let us prove that

$$
\|f\|_{\left[L^{p_{0}(\cdot)}\left(\mathbb{R}^{n}, X_{0}\right), L^{p_{1}(\cdot)}\left(\mathbb{R}^{n}, X_{1}\right)\right]_{\theta}} \leq\|f\|_{L^{p(\cdot)}\left(\mathbb{R}^{n},\left[X_{0}, X_{1}\right]_{\theta}\right)} .
$$

By the scaling argument, we see that it suffices to consider the case $\|f\|_{L^{p(\cdot)}\left(\mathbb{R}^{n},\left[X_{0}, X_{1}\right]_{\theta}\right)}=$ 1. We put for $0 \leq \operatorname{Re} z \leq 1$

$$
g(x, z)=h(x, z)\|f(x)\|_{\left[X_{0}, X_{1}\right]_{\theta}}^{\left(\frac{p(x)}{p_{1}(x)}-\frac{p(x)}{p_{0}(x)}\right)(z-\theta)},
$$

where $h \in \mathcal{F}\left(X_{0}, X_{1}\right)$ for fixed $x \in \mathbb{R}^{n}, h(x, \theta)=f(x)$ and $h(x, z)=h(y, z)$ if $x, y \in$ $A_{j}, j=1, \ldots, N$ and $z$ is defined on the strip $0 \leq \operatorname{Re} z \leq 1, h(x, z)=0$ for any $x \in \mathbb{R}^{n}-\cup_{j=1}^{N} A_{j}$ and

$$
\|h(x, i t)\|_{X_{0}},\|h(x, 1+i t)\|_{X_{1}} \leq\|f(x)\|_{\left[X_{0}, X_{1}\right]_{\theta}}+\varepsilon
$$

$\varepsilon$ is a given positive number. Using this we derive

$$
\begin{aligned}
\varrho_{L^{p_{0}(\cdot)}\left(\mathbb{R}^{n}, X_{0}\right)}(g(i t)) & =\int_{\mathbb{R}^{n}}|h(x, i t)|^{p_{0}(x)}\|f(x)\|_{\left[X_{0}, X_{1}\right]_{\theta}}^{-\left(\frac{p(x)}{p_{1}(x)}-\frac{p(x)}{p_{0}(x)}\right) \theta p_{0}(x)} d x \\
& \leq \int_{\mathbb{R}^{n}}\|f(x)\|_{\left[X_{0}, X_{1}\right]_{\theta}}^{p(x)} d x+\varepsilon^{\prime}=1+\varepsilon^{\prime} .
\end{aligned}
$$

Similarly,

$$
\varrho_{L^{p_{1}(\cdot)}\left(\mathbb{R}^{n}, X_{1}\right)}(g(1+i t)) \leq 1+\varepsilon^{\prime} .
$$

Thus

$$
\|g\|_{\mathcal{F}\left(L^{p_{0}(\cdot)}\left(\mathbb{R}^{n}, X_{0}\right), L^{p_{1}(\cdot)}\left(\mathbb{R}^{n}, X_{1}\right)\right)} \leq 1 .
$$

This and $g(\cdot, \theta)=f(\cdot)$ we obtain

$$
\|f\|_{\left[L^{p_{0}(\cdot)}\left(\mathbb{R}^{n}, X_{0}\right), L^{p_{1}(\cdot)}\left(\mathbb{R}^{n}, X_{1}\right)\right]_{\theta}} \leq 1 .
$$

Now let us prove that

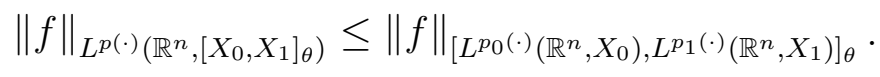


Let $f$ be a simple function of type (10). Further, let $g(x, z) \in \mathcal{F}\left(X_{0}, X_{1}\right)$ where $g(x, \theta)=f(x)$. We will prove that

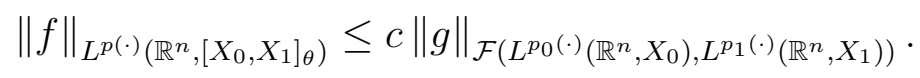

From Lemma 4.3.2 in [2] and Hölder's inequality, we obtain

$$
\begin{aligned}
\|f(x)\|_{\left[X_{0}, X_{1}\right]_{\theta}} \leq & \left(\frac{1}{1-\theta} \int_{R}\|g(x, i t)\|_{X_{0}} \mu_{0}(\theta, t) d t\right)^{1-\theta} \\
& \times\left(\frac{1}{\theta} \int_{R}\|g(x, 1+i t)\|_{X_{1}} \mu_{1}(\theta, t) d t\right)^{\theta},
\end{aligned}
$$

where $\mu_{0}(\theta, t)$ and $\mu_{1}(\theta, t)$ are the Poisson kernels for the strip $A_{0}$,

$$
\frac{1}{1-\theta} \int_{R} \mu_{0}(\theta, t) d t=\frac{1}{\theta} \int_{R} \mu_{1}(\theta, t) d t=1
$$

Using Hölder's inequality with respect to the variable $t$ we get

$$
\begin{aligned}
\|f(x)\|_{\left[X_{0}, X_{1}\right]_{\theta}} \leq & \left(\frac{1}{1-\theta} \int_{R}\|g(x, i t)\|_{X_{0}}^{p_{0}(x)} \mu_{0}(\theta, t) d t\right)^{(1-\theta) / p_{0}(x)} \\
& \times\left(\frac{1}{\theta} \int_{R}\|g(x, 1+i t)\|_{X_{1}}^{p_{1}(x)} \mu_{1}(\theta, t) d t\right)^{\theta / p_{1}(x)} .
\end{aligned}
$$

Hence, again by Hölder's inequality with respect to the variable $x$ we get

$$
\begin{aligned}
& \|f\|_{L^{p(\cdot)}\left(\mathbb{R}^{n},\left[X_{0}, X_{1}\right]_{\theta}\right)} \lesssim\left\|\left(\frac{1}{1-\theta} \int_{R}\|g(x, i t)\|_{X_{0}}^{p_{0}(\cdot)} \mu_{0}(\theta, t) d t\right)^{1 / p_{0}(\cdot)}\right\|_{p_{0}(\cdot)}^{1-\theta} \\
& \times\left\|\left(\frac{1}{\theta} \int_{R}\|g(x, 1+i t)\|_{X_{0}}^{p_{1}(\cdot)} \mu_{0}(\theta, t) d t\right)^{1 / p_{1}(\cdot)}\right\|_{p_{1}(\cdot)}^{\theta} .
\end{aligned}
$$

The first norm is bounded by

$$
\|g\|_{\mathcal{F}\left(L^{p_{0}(\cdot)}\left(\mathbb{R}^{n}, X_{0}\right), L^{p_{1}(\cdot)}\left(\mathbb{R}^{n}, X_{1}\right)\right)} \cdot
$$

Indeed,

$$
\begin{aligned}
\int_{\mathbb{R}^{n}} \frac{1}{1-\theta} \int_{R}\left\|\frac{g(x, i t)}{\|g\|_{\mathcal{F}}}\right\|_{X_{0}}^{p_{0}(x)} \mu_{0}(\theta, t) d t d x & =\frac{1}{1-\theta} \int_{R} \int_{\mathbb{R}^{n}}\left\|\frac{g(x, i t)}{\|g\|_{\mathcal{F}}}\right\|_{X_{0}}^{p_{0}(x)} d x \mu_{0}(\theta, t) d t \\
& \leq \frac{1}{1-\theta} \int_{R} \mu_{0}(\theta, t) d t=1 .
\end{aligned}
$$

Similarly for the rest term. The proof of theorem is complete.

Now we present complex interpolation of variable Triebel-Lizorkin space $F_{p(\cdot), q}^{\alpha}$. We use the so-called retraction method which allows us to reduce the problem to the interpolation of appropriate sequence spaces. We recall that a Banach space $X$ is called a retract of a Banach space $Y$ if there are linear continuous operators $R: Y \rightarrow X$ (retraction) and $S: X \rightarrow Y$ (co-retraction) such that the composition $R S$ is the identity operator in $X$.

First let us recall the Littlewood-Paley decomposition. Let $\Psi$ be a function in $\mathcal{S}\left(\mathbb{R}^{n}\right)$ satisfying $0 \leq \Psi(x) \leq 1$ for all $x \in \mathbb{R}^{n}, \Psi(x)=1$ for $|x| \leq 1$ and $\Psi(x)=0$ for 
$|x| \geq 2$. We put $\mathcal{F} \varphi_{0}(x)=\Psi(x), \mathcal{F} \varphi(x)=\Psi\left(\frac{x}{2}\right)-\Psi(x)$ and $\mathcal{F} \varphi_{v}(x)=\mathcal{F} \varphi\left(2^{1-v} x\right)$ for $v=1,2,3, \ldots$. Then $\left\{\mathcal{F} \varphi_{v}\right\}_{v \in \mathbb{N}_{0}}$ is a resolution of unity, $\sum_{v=0}^{\infty} \mathcal{F} \varphi_{v}(x)=1$ for all $x \in \mathbb{R}^{n}$. Thus we obtain the Littlewood-Paley decomposition

$$
f=\sum_{v=0}^{\infty} \varphi_{v} * f
$$

of all $f \in \mathcal{S}^{\prime}\left(\mathbb{R}^{n}\right)$ (convergence in $\mathcal{S}^{\prime}\left(\mathbb{R}^{n}\right)$ ). We define

$$
S(f)=\left(\varphi_{v} * f\right)_{v}
$$

and

$$
R\left(\left(f_{v}\right)_{v}\right)=\sum_{v=0}^{\infty} \omega_{v} * f_{v}
$$

where $\omega_{v}=\varphi_{v-1}+\varphi_{v}+\varphi_{v+1}$.

Theorem 3 Let $p \in C^{\log }$ with $1 \leq p^{-}<\infty, 1 \leq q \leq \infty$ and $\alpha \in \mathbb{R}$. Let $\left\{\mathcal{F} \varphi_{v}\right\}_{v \in \mathbb{N}_{0}}$ be a resolution of unity. Then $S$ is a co-retraction from $F_{p(\cdot), q}^{\alpha}$ into $L^{p(\cdot)}\left(\ell_{q}^{\alpha}\right)$ and $R$ is a corresponding retraction from $L^{p(\cdot)}\left(\ell_{q}^{\alpha}\right)$ onto $F_{p(\cdot), q}^{\alpha}$.

Proof. The convergence of the series (11) in $\mathcal{S}^{\prime}\left(\mathbb{R}^{n}\right)$ can be obtained by Lemma 4.5 of [1], where one has to take into consideration $L^{p(\cdot)}\left(\ell_{q}^{\alpha}\right) \hookrightarrow \ell_{\infty}^{\alpha}\left(L^{p(\cdot)}\right)$. Clearly $R$ is a bounded linear operator from $F_{p(\cdot), q}^{\alpha}$ into $L^{p(\cdot)}\left(\ell_{q}^{\alpha}\right)$. Moreover, $R S$ is the identity operator in $F_{p(\cdot), q}^{\alpha}$. Using the support properties of $\mathcal{F} \varphi_{v}$ and $\mathcal{F} \omega_{v}$,

$$
\begin{aligned}
\varphi_{v} * R\left(\left(f_{i}\right)_{i}\right) & =\sum_{i=v-2}^{v+2} \varphi_{v} * \omega_{i} * f_{i} \\
& =\sum_{k=-2}^{2} \varphi_{v} * \omega_{k+v} * f_{k+v}, \quad v=0,1, \ldots .
\end{aligned}
$$

We can estimate $\left|\varphi_{v} * \omega_{k+v} * f_{k+v}\right|$ by

$$
\left(\eta_{v} *\left|f_{k+v}\right|^{t}\right)^{1 / t}, \quad 0<t<\min \left(p^{-}, q\right),
$$

see [5]. Applying Lemma 2,

$$
\begin{aligned}
\left\|R\left(\left(f_{i}\right)_{i}\right)\right\|_{F_{p(\cdot), q}^{\alpha}} & \lesssim \sum_{k=-2}^{2}\left\|\left(2^{v \alpha} f_{k+v}\right)_{v \geq 0}\right\|_{L^{p(\cdot)}\left(\ell_{q}\right)} \\
& \lesssim\left\|\left(2^{v \alpha} f_{v}\right)_{v \geq 0}\right\|_{L^{p(\cdot)}\left(\ell_{q}\right)} .
\end{aligned}
$$

The proof of theorem is complete.

Now we are ready to formulate the main statement of this subsection.

Theorem 4 Let $0<\theta<1$. Let $p_{0}, p_{1} \in C^{\log }$ with $1 \leq p_{0}^{+}, p_{1}^{+}<\infty, 1 \leq q_{0}, q_{1}<\infty$ and $\alpha_{0}, \alpha_{1} \in \mathbb{R}$. We put

$$
\frac{1}{p(\cdot)}:=\frac{1-\theta}{p_{0}(\cdot)}+\frac{\theta}{p_{1}(\cdot)}, \frac{1}{q}:=\frac{1-\theta}{q_{0}}+\frac{\theta}{q_{1}} \text { and } \alpha:=(1-\theta) \alpha_{0}+\theta \alpha_{1} .
$$

Then

$$
\left[F_{p_{0}(\cdot), q_{0}}^{\alpha_{0}}, F_{p_{1}(\cdot), q_{1}}^{\alpha_{1}}\right]_{\theta}=F_{p(\cdot), q}^{\alpha}
$$

holds in the sense of equivalent norms. 
Proof. The embedding (86) shows that $\left(F_{p_{0}(\cdot), q_{0}}^{\alpha_{0}}, F_{p_{1}(\cdot), q_{1}}^{\alpha_{1}}\right)$ is an interpolation couple. By Theorem 3 ,

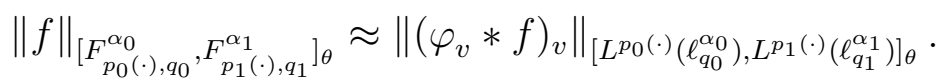

Now by Theorem 2 the left-hand side is equivalent to $\left\|\left(\varphi_{v} * f\right)_{v}\right\|_{L^{p(\cdot)}\left(\ell_{q}^{\alpha}\right)}$.

\subsection{Complex interpolation for the spaces $F_{p(\cdot), p(\cdot)}^{\alpha(\cdot)}$ and some lim- iting cases}

In this subsection we present interpolation results in $F_{p(\cdot), p(\cdot)}^{\alpha(\cdot)}$ spaces and some consequences,. We follow the approach of Frazier and Jawerth [10], see also [16] and [19]. We start by defining the Calderón product of two Banach lattices. Let $(\mathcal{A}, S, \mu)$ be a $\sigma$-finite measure space and let $\mathfrak{M}$ be the class of all complex-valued, $\mu$-measurable functions on $\mathcal{A}$. Then a Banach space $X \subset \mathfrak{M}$ is called a Banach lattice of functions if for every $f \in X$ and $g \in \mathfrak{M}$ with $|g(x)| \leq|f(x)|$ for $\mu$-a.e. $x \in X$ one has $g \in X$ and $\|g\|_{X} \leq\|f\|_{X}$

Definition 5 Let $(\mathcal{A}, S, \mu)$ be a $\sigma$-finite measure space and let $\mathfrak{M}$ be the class of all complex-valued, $\mu$-measurable functions on $\mathcal{A}$. Suppose that $X_{0}$ and $X_{1}$ are Banach lattices on $\mathfrak{M}$. Given $0<\theta<1$, define the Calderón product $X_{0}^{1-\theta} \cdot X_{1}^{\theta}$ as the collection of all functions $f \in \mathfrak{M}$ satisfying

$$
\|f\|_{X_{0}^{1-\theta} \cdot X_{1}^{\theta}}:=\inf \left\{\|g\|_{X_{0}}^{1-\theta}\|h\|_{X_{1}}^{\theta}:|f| \leq|g|^{1-\theta}|h|^{\theta}, \mu \text {-a.e., }\|g\|_{X_{0}} \leq 1,\|h\|_{X_{1}} \leq 1\right\} .
$$

Remark 1 Calderón products have been introduced by Calderón [3] (in a little bit different form which coincides with the above one). Further properties we refer to, Frazier and Jawerth [10], Mendez and Mitrea [13], Kalton, Mayboroda and Mitrea [11] and Yang, Yuan and Zhuo [19].

We need a few useful properties, see [19].

Lemma 5 Let $(\mathcal{A}, S, \mu)$ be a $\sigma$-finite measure space and let $\mathfrak{M}$ be the class of all complex-valued, $\mu$-measurable functions on $\mathcal{A}$. Suppose that $X_{0}$ and $X_{1}$ are Banach lattices on $\mathfrak{M}$. Let $0<\theta<1$.

(i) Then the Calderón product $X_{0}^{1-\theta} \cdot X_{1}^{\theta}$ is a Banach space.

(ii) Define the Calderón product $\widehat{X_{0}^{1-\theta} \cdot X_{1}^{\theta}}$ as the collection of all functions $f \in \mathfrak{M}$ satisfying

$$
\|f\|_{X_{0}^{1-\theta} \cdot X_{1}^{\theta}}:=\inf \left\{M>0:|f| \leq M|g|^{1-\theta}|h|^{\theta},\|g\|_{X_{0}} \leq 1,\|h\|_{X_{1}} \leq 1\right\} .
$$

Then $\widetilde{X_{0}^{1-\theta} \cdot X_{1}^{\theta}}=X_{0}^{1-\theta} \cdot X_{1}^{\theta}$ follows with equality of norms.

Now we turn to the investigation of the Calderón products of the sequence spaces $f_{p(\cdot), p(\cdot)}^{\alpha(\cdot)}$. 
Theorem 5 Let $0<\theta<1$. Let $p_{0}, p_{1} \in C^{\log }$ with $1 \leq p_{0}^{+}, p_{1}^{+}<\infty$ and $\alpha_{0}, \alpha_{1} \in C_{\mathrm{loc}}^{\log }$. We put

$$
\frac{1}{p(\cdot)}:=\frac{1-\theta}{p_{0}(\cdot)}+\frac{\theta}{p_{1}(\cdot)} \text { and } \alpha(\cdot):=(1-\theta) \alpha_{0}(\cdot)+\theta \alpha_{1}(\cdot) \text {. }
$$

Then

$$
\left(f_{p_{0}(\cdot), p_{0}(\cdot)}^{\alpha_{0}(\cdot)}\right)^{1-\theta}\left(f_{p_{1}(\cdot), p_{1}(\cdot)}^{\alpha_{1}(\cdot)}\right)^{\theta}=f_{p(\cdot), p(\cdot)}^{\alpha(\cdot)}
$$

holds in the sense of equivalent norms.

\section{Proof.}

Step 1. We shall prove

$$
\left(f_{p_{0}(\cdot), p_{0}(\cdot)}^{\alpha_{0}(\cdot)}\right)^{1-\theta}\left(f_{p_{1}(\cdot), p_{1}(\cdot)}^{\alpha_{1}(\cdot)}\right)^{\theta} \hookrightarrow f_{p(\cdot), p(\cdot)}^{\alpha(\cdot)}
$$

We suppose, that sequences $\lambda:=\left(\lambda_{j, m}\right)_{j, m}, \lambda^{i}:=\left(\lambda_{j, m}^{i}\right)_{j, m}, i=0,1$, are given and that

$$
\left|\lambda_{j, m}\right| \leq\left|\lambda_{j, m}^{0}\right|^{1-\theta}\left|\lambda_{j, m}^{1}\right|^{\theta}
$$

holds for all $j \in \mathbb{N}_{0}$ and $m \in \mathbb{Z}^{n}$. Let

$$
g(x):=\left(\sum_{j=0}^{\infty} \sum_{m \in \mathbb{Z}^{n}} 2^{j(\alpha(x)+n / 2) p(x)}\left|\lambda_{j, m}\right|^{p(x)} \chi_{j, m}(x)\right)^{1 / p(x)} .
$$

Since,

$$
\begin{aligned}
& 2^{j(\alpha(x)+n / 2) p(x)}\left|\lambda_{j, m}\right|^{p(x)} \chi_{j, m}(x) \\
= & \left(2^{j\left(\alpha_{0}(x)+n / 2\right)}\left|\lambda_{j, m}\right| \chi_{j, m}(x)\right)^{p(x)(1-\theta)}\left(2^{j\left(\alpha_{1}(x)+n / 2\right)}\left|\lambda_{j, m}\right| \chi_{j, m}(x)\right)^{p(x) \theta},
\end{aligned}
$$

then, Hölder's inequality implies that $g(x)$ can be estimated by

$$
\begin{aligned}
& \left(\sum_{j=0}^{\infty} \sum_{m \in \mathbb{Z}^{n}}\left(2^{j\left(\alpha_{0}(x)+n / 2\right)}\left|\lambda_{j, m}\right| \chi_{j, m}(x)\right)^{p_{0}(x)}\right)^{(1-\theta) / p_{0}(x)} \\
& \times\left(\sum_{j=0}^{\infty} \sum_{m \in \mathbb{Z}^{n}}\left(2^{j\left(\alpha_{1}(x)+n / 2\right)}\left|\lambda_{j, m}\right| \chi_{j, m}(x)\right)^{p_{1}(x)}\right)^{\theta / p_{1}(x)} .
\end{aligned}
$$

Apply Hölder's inequality again but with conjugate indices $\frac{p_{0}(\cdot)}{(1-\theta)}$ and $\frac{p_{1}(\cdot)}{\theta}$, we obtain

$$
\|\lambda\|_{f_{p(\cdot), p(\cdot)}^{\alpha(\cdot)}} \leq\left\|\lambda^{0}\right\|_{f_{p_{0}(\cdot), p_{0}(\cdot)}^{\alpha_{0}(\cdot)}}^{1-\theta}\left\|\lambda^{1}\right\|_{f_{p_{1}(\cdot), p_{1}(\cdot)}^{\alpha_{1}(\cdot)}}^{\theta} .
$$

Step 2. Now we turn to the proof of

$$
f_{p(\cdot), p(\cdot)}^{\alpha(\cdot)} \hookrightarrow\left(f_{p_{0}(\cdot), p_{0}(\cdot)}^{\alpha_{0}(\cdot)}\right)^{1-\theta}\left(f_{p_{1}(\cdot), p_{1}(\cdot)}^{\alpha_{1}(\cdot)}\right)^{\theta}
$$

We will use Lemma 5. Let the sequence $\lambda \in f_{p(\cdot), p(\cdot)}^{\alpha(\cdot)}$ be given with

$$
\|\lambda\|_{f_{p(\cdot), p(\cdot)}^{\alpha(\cdot)}} \neq 0 .
$$


We have to find sequences $\lambda^{0}$ and $\lambda^{1}$ such that $\left|\lambda_{j, m}\right| \leq M\left|\lambda_{j, m}^{0}\right|^{1-\theta}\left|\lambda_{j, m}^{1}\right|^{\theta}$ for every $j \in \mathbb{N}_{0}, m \in \mathbb{Z}^{n}$ and

$$
\left\|\lambda^{0}\right\|_{f_{p_{0}(\cdot), p_{0}(\cdot)}^{\alpha_{0}(\cdot)}} \lesssim 1 \text { and }\left\|\lambda^{1}\right\|_{f_{p_{1}(\cdot), p_{1}(\cdot)}^{\alpha_{1}(\cdot)}} \lesssim 1
$$

with some constant $c$ independent of $\lambda$. We follow ideas of the proof of Theorem 8.2 in Frazier and Jawerth [10], see also Sickel, Skrzypczak and Vybíral [16]. Set

$$
u(\cdot):=p(\cdot) \theta\left(\frac{\alpha_{1}(\cdot)}{p_{0}(\cdot)}-\frac{\alpha_{0}(\cdot)}{p_{1}(\cdot)}\right)+\frac{n}{2}\left(\frac{p(\cdot)}{p_{0}(\cdot)}-1\right)
$$

and

We put

$$
v(\cdot):=p(\cdot)(1-\theta)\left(\frac{\alpha_{0}(\cdot)}{p_{1}(\cdot)}-\frac{\alpha_{1}(\cdot)}{p_{0}(\cdot)}\right)+\frac{n}{2}\left(\frac{p(\cdot)}{p_{1}(\cdot)}-1\right) .
$$

$$
\lambda_{j, m}^{0}:=2^{j u\left(x_{j, m}\right)}\left(\frac{\left|\lambda_{j, m}\right|}{\|\lambda\|_{f_{p(\cdot), p(\cdot)}^{\alpha(\cdot)}}}\right)^{p\left(x_{j, m}\right) / p_{0}\left(x_{j, m}\right)}, \quad x_{j, m}=2^{-j} m
$$

Also, set

$$
\lambda_{j, m}^{1}:=2^{j v\left(x_{j, m}\right)}\left(\frac{\left|\lambda_{j, m}\right|}{\|\lambda\|_{f_{p(\cdot), p(\cdot)}^{\alpha(\cdot)}}}\right)^{p\left(x_{j, m}\right) / p_{1}\left(x_{j, m}\right)} .
$$

Observe that

$$
\left|\lambda_{j, m}\right| \leq\|\lambda\|_{f_{p(\cdot), p(\cdot)}^{\alpha()}}\left(\lambda_{j, m}^{0}\right)^{1-\theta}\left(\lambda_{j, m}^{1}\right)^{\theta}
$$

which holds now for all pairs $(j, m)$.

Estimation of $\left\|\lambda^{0}\right\|_{f_{p_{0}(\cdot), p_{0}(\cdot)}^{\alpha_{0}(\cdot)}}$. Set

$$
I(\cdot):=\sum_{j=0}^{\infty} \sum_{m \in \mathbb{Z}^{n}}\left(2^{j\left(\alpha_{0}(\cdot)+\frac{n}{2}\right)}\left|\lambda_{j, m}^{0}\right| \chi_{j, m}\right)^{p_{0}(\cdot)} .
$$

We use the local log-Hölder continuity of $\alpha_{0}, \alpha_{1}, p_{0}$ and $p_{1}$ to show that

$$
2^{j u(x)} \leq c 2^{j u(y)} \quad \text { and } \quad 2^{j\left(\alpha(x)-\frac{n}{p(x)}\right)} \leq c 2^{j\left(\alpha(y)-\frac{n}{p(y)}\right)}, \quad x, y \in Q_{j, m},
$$

where $c>0$ is independent of $m \in \mathbb{Z}^{n}$ and $j \in \mathbb{N}_{0}$. Now $\left(\frac{\left|\lambda_{j, m}\right|}{\|\lambda\|_{f_{p(\cdot), p(\cdot)}^{\alpha(\cdot)}}}\right)^{\frac{p\left(x_{j, m}\right)}{p_{0}\left(x_{j, m}\right)}}$ can be rewritten us

$$
\left(2^{j\left(\alpha\left(x_{j, m}\right)+\frac{n}{2}-\frac{n}{p\left(x_{j, m}\right)}\right)} \frac{\left|\lambda_{j, m}\right|}{\|\lambda\|_{f_{p(\cdot), p(\cdot)}^{\alpha(\cdot)}}}\right)^{\frac{p\left(x_{j, m}\right)}{p_{0}\left(x_{j, m}\right)}} 2^{-j\left(\alpha\left(x_{j, m}\right)+\frac{n}{2}-\frac{n}{p\left(x_{j, m}\right)}\right) \frac{p\left(x_{j, m}\right)}{p_{0}\left(x_{j, m}\right)}} .
$$

After applying Lemma 3, we get

$$
2^{j\left(\alpha\left(x_{j, m}\right)+\frac{n}{2}-\frac{n}{p\left(x_{j, m}\right)}\right)} \frac{\left|\lambda_{j, m}\right|}{\|\lambda\|_{f_{p(\cdot), p(\cdot)}^{\alpha()}}} \lesssim 1 .
$$

Observe that

$$
\begin{aligned}
2^{j\left(\alpha\left(x_{j, m}\right)+\frac{n}{2}-\frac{n}{p\left(x_{j, m}\right)}\right)} \frac{\left|\lambda_{j, m}\right|}{\|\lambda\|_{f_{p(\cdot), p(\cdot)}^{\alpha(\cdot)}}} & =\frac{1}{\left|Q_{j, m}\right|} \int_{Q_{j, m}} 2^{j\left(\alpha\left(x_{j, m}\right)+\frac{n}{2}-\frac{n}{p\left(x_{j, m}\right)}\right)} \frac{\left|\lambda_{j, m}\right|}{\|\lambda\|_{f_{p(\cdot), p(\cdot)}^{\alpha(\cdot)}}} d y \\
& \lesssim \frac{1}{\left|Q_{j, m}\right|} \int_{Q_{j, m}} 2^{j\left(\alpha(y)+\frac{n}{2}-\frac{n}{p(y)}\right)} \frac{\left|\lambda_{j, m}\right|}{\|\lambda\|_{f_{p(\cdot), p(\cdot)}^{\alpha(\cdot)}}} d y
\end{aligned}
$$


for any $m \in \mathbb{Z}^{n}$ and any $j \in \mathbb{N}_{0}$. Taking the $\frac{\sigma\left(x_{j, m}\right) p\left(x_{j, m}\right)}{p_{0}\left(x_{j, m}\right)}$-power, with $\frac{p_{0}(\cdot)}{p(\cdot)}<\sigma(\cdot)<1$ and $\sigma \in C^{\text {log }}$, by (17) we can apply Lemma 4 and obtain that

$$
\begin{aligned}
& \left(2^{j\left(\alpha\left(x_{j, m}\right)+\frac{n}{2}-\frac{n}{p\left(x_{j, m}\right)}\right)} \frac{\left|\lambda_{j, m}\right|}{\|\lambda\|_{f_{p(\cdot), p(\cdot)}^{\alpha(\cdot)}}}\right)^{\frac{\sigma\left(x_{j, m}\right) p\left(x_{j, m}\right)}{p_{0}\left(x_{j, m}\right)}} \\
\lesssim & \frac{1}{\left|Q_{j, m}\right|} \int_{Q_{j, m}}\left(2^{j\left(\alpha(y)+\frac{n}{2}-\frac{n}{p(y)}\right)} \frac{\left|\lambda_{j, m}\right|}{\|\lambda\|_{f_{p(\cdot), p(\cdot)}^{\alpha(\cdot)}}}\right)^{\frac{\sigma(y) p(y)}{p_{0}(y)}} d y+2^{-j n h} \eta_{0, h}\left(x_{j, m}\right) .
\end{aligned}
$$

Applying again Lemma 3, the last term with $\frac{1}{\sigma\left(x_{j, m}\right)}$-power is bounded by

$$
\begin{aligned}
& \frac{2^{\frac{1}{\sigma^{-}}-1}}{\left|Q_{j, m}\right|} \int_{Q_{j, m}}\left(2^{j\left(\alpha(y)+\frac{n}{2}-\frac{n}{p(y)}\right)} \frac{\left|\lambda_{j, m}\right|}{\|\lambda\|_{f_{p(\cdot), p(\cdot)}^{\alpha(\cdot)}}}\right)^{\frac{p(y)}{p_{0}(y)}} d y+2^{\frac{1}{\sigma^{-}-j n h+1} \eta_{0, h}\left(x_{j, m}\right)} \\
\lesssim & \eta_{j, \rho} *\left(2^{j\left(\alpha(\cdot)+\frac{n}{2}-\frac{n}{p(\cdot)}\right)} \frac{\left|\lambda_{j, m}\right|}{\|\lambda\|_{f_{p(\cdot), p(\cdot)}^{\alpha(\cdot)}}} \chi_{Q_{j, m}}\right)^{\frac{p(\cdot)}{p_{0}(\cdot)}}(x)+2^{-j n h} \eta_{0, h}(x)
\end{aligned}
$$

for any $h, \rho>0$ and any $x \in Q_{j, m}$. Applying the second estimate of (16), the term $\left(\frac{\left|\lambda_{j, m}\right|}{\|\lambda\|_{f_{p(\cdot), p(\cdot)}^{\alpha(\cdot)}}}\right)^{\frac{p\left(x_{j, m}\right)}{p_{0}\left(x_{j, m}\right)}}$ can be estimated by

$$
c \eta_{j, \rho_{1}} *\left(\frac{\left|\lambda_{j, m}\right|}{\|\lambda\|_{f_{p(\cdot), p(\cdot)}^{\alpha(\cdot)}}} \chi_{Q_{j, m}}\right)^{\frac{p(\cdot)}{p_{0}(\cdot)}}(x)+c 2^{-j(n h+d)} \eta_{0, h}(x)
$$

where

$$
d=\left(\left(\alpha+\frac{n}{2}-\frac{n}{p}\right) \frac{p}{p_{0}}\right)^{-} \text {and } \rho_{1}=\rho-c_{\log }\left(\left(\alpha+\frac{n}{2}-\frac{n}{p}\right) \frac{p}{p_{0}}\right) .
$$

From the estimations above, for any $x \in Q_{j, m}$

$$
2^{j\left(\alpha_{0}(x)+\frac{n}{2}\right)}\left|\lambda_{j, m}^{0}\right| \lesssim \eta_{j, \rho_{2}} * \digamma_{j, m}(x)+2^{-j\left(n h+d-\left(u^{+}+\alpha_{0}^{+}+\frac{n}{2}\right)\right)} \eta_{0, h}(x),
$$

where the implicit positive constant not depending on $x, m$ and $j$, with

$$
\digamma_{j, m}=2^{j\left(\alpha_{0}(\cdot)+\frac{n}{2}\right)+j u(\cdot)}\left(\frac{\left|\lambda_{j, m}\right|}{\|\lambda\|_{f_{p(\cdot), p(\cdot)}^{\alpha(\cdot)}}}\right)^{\frac{p(\cdot)}{p_{0}(\cdot)}} \chi_{Q_{j, m}}
$$

where

$$
\rho_{2}=\rho_{1}-c_{\log }\left(\alpha_{0}\right)-c_{\log }(u)
$$

Hence $\left\|\left(I^{p_{0}(\cdot)}(\cdot)\right)^{1 / p_{0}(\cdot)}\right\|_{p_{0}(\cdot)}$ is bounded by

$c\left\|\left(\sum_{j=0}^{\infty} \sum_{m \in \mathbb{Z}^{n}}\left(\eta_{j, \rho_{2}} * \digamma_{j, m}\right)^{p_{0}(\cdot)}\right)^{1 / p_{0}(\cdot)}\right\|_{p_{0}(\cdot)}+c\left\|\eta_{0, h}\right\|_{p_{0}(\cdot)}\left(\sum_{j=0}^{\infty} 2^{-j\left(n h+d-\left(u^{+}+\alpha_{0}^{+}+\frac{n}{2}\right)\right) p_{0}^{-}}\right)^{\frac{1}{p_{0}^{-}}}$

where in the second estimate we used the embedding $L^{p(\cdot)}\left(\ell^{p^{-}}\right) \hookrightarrow L^{p(\cdot)}\left(\ell^{p(\cdot)}\right)$. By taking $h$ large enough such that $h>\left(\alpha_{0}^{+}+u^{+}+\frac{n}{2}\right) / n-\frac{d}{n}$ the second term is bounded. 
Taking $\rho_{2}$ large enough and applying Lemma 2 to estimate the first expression by

$$
\begin{aligned}
& c\left\|\left(\sum_{j=0}^{\infty} \sum_{m \in \mathbb{Z}^{n}} \digamma_{j, m}^{p_{0}(\cdot)}\right)^{1 / p_{0}(\cdot)}\right\|_{p_{0}(\cdot)} \\
= & c\left\|\left(\sum_{j=0}^{\infty} \sum_{m \in \mathbb{Z}^{n}} 2^{j\left(\alpha(\cdot)+\frac{n}{2}\right) p(\cdot)}\left(\frac{\left|\lambda_{j, m}\right|}{\|\lambda\|_{f_{p(\cdot), p(\cdot)}^{\alpha(\cdot)}}}\right)^{q(\cdot)} \chi_{Q_{j, m}}\right)^{1 / p_{0}(\cdot)}\right\|_{p_{0}(\cdot)},
\end{aligned}
$$

since $u(\cdot)+\alpha_{0}(\cdot)=\alpha(\cdot) \frac{p(\cdot)}{p_{0}(\cdot)}+\frac{n}{2}\left(\frac{p(\cdot)}{p_{0}(\cdot)}-1\right)$. Therefore, $\left\|\left(I^{p_{0}(\cdot)}(\cdot)\right)^{1 / p_{0}(\cdot)}\right\|_{p_{0}(\cdot)}$ is bounded by

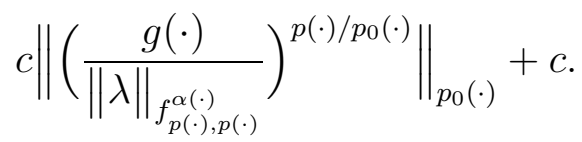

This term is bounded, since

$$
\int_{\mathbb{R}^{n}}\left(\frac{g(x)}{\|\lambda\|_{f_{p(\cdot), p(\cdot)}^{\alpha(\cdot)}}}\right)^{p(x)} d x \leq 1
$$

Estimation of $\left\|\lambda^{1}\right\|_{f_{p_{1}(\cdot), p_{1}(\cdot)}^{\alpha_{1}(\cdot)}}$. Replacing $\alpha_{0}, p_{0}$ and $u$ by $\alpha_{1}, p_{1}$ and $v$, respectively and this leads to the desired inequality.

Notice that this theorem can be generalized to the case $0<p_{0}^{+}, p_{1}^{+}<\infty$.

Theorem 6 Let $0<\theta<1$ and $1 \leq q_{0}, q_{1}<\infty$. Let $p_{0} \in C^{\log }$ with $1 \leq p_{0}^{+}<\infty$ and $\alpha_{0}, \alpha_{1} \in C_{\mathrm{loc}}^{\mathrm{log}}$. We put

$$
\frac{1}{p(\cdot)}:=\frac{1-\theta}{p_{0}(\cdot)}, \frac{1}{q}:=\frac{1-\theta}{q_{0}}+\frac{\theta}{q_{1}} \text { and } \alpha(\cdot):=(1-\theta) \alpha_{0}(\cdot)+\theta \alpha_{1}(\cdot) \text {. }
$$

Then

$$
\left(f_{p_{0}(\cdot), q_{0}}^{\alpha_{0}(\cdot)}\right)^{1-\theta}\left(f_{\infty, q_{1}}^{\alpha_{1}(\cdot)}\right)^{\theta}=f_{p(\cdot), q}^{\alpha(\cdot)}
$$

holds in the sense of equivalent norms.

Proof.

Step 1. We shall prove

$$
\left(f_{p_{0}(\cdot), q_{0}}^{\alpha_{0}(\cdot)}\right)^{1-\theta}\left(f_{\infty, q_{1}}^{\alpha_{1}(\cdot)}\right)^{\theta} \hookrightarrow f_{p(\cdot), q}^{\alpha(\cdot)}
$$

We suppose, that sequences $\lambda:=\left(\lambda_{j, m}\right)_{j, m}, \lambda^{i}:=\left(\lambda_{j, m}^{i}\right)_{j, m}, i=0,1$, are given and that

$$
\left|\lambda_{j, m}\right| \leq\left|\lambda_{j, m}^{0}\right|^{1-\theta}\left|\lambda_{j, m}^{1}\right|^{\theta}
$$

holds for all $j \in \mathbb{N}_{0}$ and $m \in \mathbb{Z}^{n}$. In (13) we replace $p(x)$ by $q$ and $\chi_{j, m}$ by $\chi_{E_{Q_{j, m}}}$, with $E_{Q_{j, m}} \subset Q_{j, m}$ and $\left|E_{Q_{j, m}}\right|>\left|Q_{j, m}\right| / 2$, we obtain (14) with $q_{0}$ and $q_{1}$ in place of $p_{0}(x)$ and $p_{0}(x)$, respectively, and $\chi_{E_{Q_{j, m}}}$ in place of $\chi_{j, m}$. Estimate the second factor by its $L^{\infty}$-norm and using Propositions 1 we get $\|\lambda\|_{f_{p(\cdot), q}^{\alpha(\cdot)}} \leq\left\|\lambda^{0}\right\|_{f_{p_{0}(\cdot), q_{0}}^{\alpha_{0}(\cdot)}}^{1-\theta}\left\|\lambda^{1}\right\|_{f_{\infty, q_{1}}^{\alpha_{1}(\cdot)}}^{\theta}$.

Step 2. We prove

$$
f_{p(\cdot), q}^{\alpha(\cdot)} \hookrightarrow\left(f_{p_{0}(\cdot), q_{0}}^{\alpha_{0}(\cdot)}\right)^{1-\theta}\left(f_{\infty, q_{1}}^{\alpha_{1}(\cdot)}\right)^{\theta} .
$$


Let the sequence $\lambda \in f_{p(\cdot), q}^{\alpha(\cdot)}$ be given with

$$
\|\lambda\|_{f_{p(\cdot), q}^{\alpha(\cdot)}} \neq 0
$$

Let $\delta=-\frac{q}{q_{1}}$ and $\gamma(\cdot):=\frac{p(\cdot)}{p_{0}(\cdot)}-\frac{q}{q_{0}}$. Observe that $\gamma$ is a constant function. We follow ideas of the proof of Theorem 8.2 in Frazier and Jawerth [10]. Set

$$
A_{\ell, \gamma}:=\left\{x \in \mathbb{R}^{n}:\left(\frac{g(x)}{\|\lambda\|_{f_{p(\cdot), q}^{\alpha(\cdot)}}}\right)^{\gamma}>2^{\ell}\right\}
$$

with $\ell \in \mathbb{Z}$. Obviously $A_{\ell+1, \gamma} \subset A_{\ell, \gamma}$ for any $\ell \in \mathbb{Z}$. Now we introduce a (partial) decomposition of $\mathbb{N}_{0} \times \mathbb{Z}^{n}$ by taking

$$
C_{\ell}^{\gamma}:=\left\{(j, m):\left|Q_{j, m} \cap A_{\ell, \gamma}\right|>\frac{\left|Q_{j, m}\right|}{2} \text { and }\left|Q_{j, m} \cap A_{\ell+1, \gamma}\right| \leq \frac{\left|Q_{j, m}\right|}{2}\right\}, \quad \ell \in \mathbb{Z} .
$$

The sets $C_{\ell}^{\gamma}$ are pairwise disjoint, i.e., $C_{\ell}^{\gamma} \cap C_{v}^{\gamma}=\emptyset$ if $\ell \neq v$. Let us prove that $\lambda_{j, m}=0$ holds for all tuples $(j, m) \notin \cup_{\ell} C_{\ell}^{\gamma}$. Let us consider one such tuple $\left(j_{0}, m_{0}\right)$ and let us choose $\ell_{0} \in \mathbb{Z}$ arbitrary. First suppose that $\left(j_{0}, m_{0}\right) \notin C_{\ell_{0}}^{\gamma}$, then either

$$
\left|Q_{j_{0}, m_{0}} \cap A_{\ell_{0}, \gamma}\right| \leq \frac{\left|Q_{j_{0}, m_{0}}\right|}{2} \text { or }\left|Q_{j_{0}, m_{0}} \cap A_{\ell_{0}+1, \gamma}\right|>\frac{\left|Q_{j_{0}, m_{0}}\right|}{2} .
$$

Let us assume for the moment that the second condition is satisfied. By induction on $\ell$ it follows

$$
\left|Q_{j_{0}, m_{0}} \cap A_{\ell+1, \gamma}\right|>\frac{\left|Q_{j_{0}, m_{0}}\right|}{2} \text { for all } \ell \geq \ell_{0} .
$$

Let $D:=\cap_{\ell \geq \ell_{0}} Q_{j_{0}, m_{0}} \cap A_{\ell+1, \gamma}$. The family $\left\{Q_{j_{0}, m_{0}} \cap A_{\ell, \gamma}\right\}_{\ell}$ is a decreasing family of sets, i.e., $Q_{j_{0}, m_{0}} \cap A_{\ell+1, \gamma} \subset Q_{j_{0}, m_{0}} \cap A_{\ell, \gamma}$. Therefore, in view of (19)), the measure of the set $D$ is larger than or equal to $\frac{\left|Q_{j_{0}, m_{0} \mid}\right|}{2}$. Hence $\varrho_{p(\cdot)}\left(\frac{g}{\|\lambda\|_{f_{p(\cdot), q}^{\alpha(\cdot)}}}\right)$ is greater than or equal to

$$
\int_{\mathbb{R}^{n}}\left(\frac{g(x)}{\|\lambda\|_{f_{p(\cdot), q}^{\alpha(\cdot)}}}\right)^{p(x)} \chi_{Q_{j_{0}, m_{0}} \cap A_{\ell+1, \gamma}}(x) d x \geq 2^{\ell \frac{p^{-}}{\gamma}}|D|, \quad \ell \geq \max \left(\ell_{0}, 0\right) .
$$

Now $\varrho_{p(\cdot)}\left(\frac{g}{\|\lambda\|_{f_{p(\cdot), q}^{\alpha(\cdot)}}}\right)$ is finite, since $\lambda \in f_{p(\cdot), q}^{\alpha(\cdot)}$, letting $\ell$ tend to infinity and using that $|D| \geq \frac{\left|Q_{j_{0}, m_{0}}\right|}{2}$, we get a contradiction. Hence, we have to turn in (18) to the situation where the first condition is satisfied. We claim,

$$
\left|Q_{j_{0}, m_{0}} \cap A_{\ell, \gamma}\right| \leq \frac{\left|Q_{j_{0}, m_{0}}\right|}{2} \text { for all } \ell \in \mathbb{Z}
$$

Obviously this yields

$$
\left|Q_{j_{0}, m_{0}} \cap A_{\ell, \gamma}^{c}\right|>\frac{\left|Q_{j_{0}, m_{0}}\right|}{2} \text { for all } \ell \in \mathbb{Z}
$$

again this follows by induction on $\ell$ using $\left(j_{0}, m_{0}\right) \notin \cup_{\ell} C_{\ell}^{\gamma}$. Set $E=\cap_{\ell \geq \max \left(0,-\ell_{0}\right)} Q_{j_{0}, m_{0}} \cap$ $A_{-\ell, \gamma}^{c}=\bigcap_{\ell \geq \max \left(0,-\ell_{0}\right)} h_{\ell}$. The family $\left\{h_{\ell}\right\}_{\ell}$ is a decreasing family of sets, i.e., $h_{\ell+1} \subset h_{\ell}$. 
Therefore, in view of (20) , the measure of the set $E$ is larger than or equal to $\frac{\left|Q_{j_{0}, m_{0}}\right|}{2}$. By selecting a point $x \in E$ we obtain

$$
2^{j_{0} \alpha(x)}\left|\lambda_{j_{0}, m_{0}}\right| \leq g(x) \leq\|\lambda\|_{f_{p(\cdot), q}^{\alpha(\cdot)}} 2^{-\ell / \gamma}
$$

Now, for $\ell$ tending to $+\infty$ the claim, namely $\lambda_{j_{0}, m_{0}}=0$, follows. We put, as in the proof of Theorem 5,

$$
u(\cdot):=q \theta\left(\frac{\alpha_{1}(\cdot)}{q_{0}}-\frac{\alpha_{0}(\cdot)}{q_{1}}\right)+\frac{n}{2}\left(\frac{q}{q_{0}}-1\right)
$$

and

$$
v(\cdot):=q(1-\theta)\left(\frac{\alpha_{0}(\cdot)}{q_{1}}-\frac{\alpha_{1}(\cdot)}{q_{0}}\right)+\frac{n}{2}\left(\frac{q}{q_{1}}-1\right) .
$$

If $(j, m) \notin \cup_{\ell \in \mathbb{Z}} C_{\ell}^{\gamma}$, then we define $\lambda_{j, m}^{0}=\lambda_{j, m}^{1}=0$. Let $(j, m) \in C_{\ell}^{\gamma}$. We set

$$
\lambda_{j, m}^{0}:=\lambda_{j, m, u, q_{0}}^{0, \frac{\delta}{\gamma}}:=2^{\ell+j u\left(x_{j, m}\right)}\left(\frac{\left|\lambda_{j, m}\right|}{\|\lambda\|_{f_{p(\cdot), q}^{\alpha(\cdot)}}}\right)^{q / q_{0}} .
$$

Also, set

$$
\lambda_{j, m}^{1}:=\lambda_{j, m, v, q_{1}}^{1, \frac{\delta}{\gamma}}:=2^{\ell \frac{\delta}{\gamma}+j v\left(x_{j, m}\right)}\left(\frac{\left|\lambda_{j, m}\right|}{\|\lambda\|_{f_{p(\cdot), q}^{\alpha(\cdot)}}}\right)^{q / q_{1}} .
$$

Observe that

$$
\begin{aligned}
\left|\lambda_{j, m}\right| & \leq\|\lambda\|_{f_{p(\cdot), q(\cdot)}^{\alpha(\cdot)}} 2^{-\ell\left(1-\theta+\frac{\delta}{\gamma} \theta\right)} 2^{-j\left(u\left(x_{j, m}\right)(1-\theta)+v\left(x_{j, m}\right) \theta\right)}\left(\lambda_{j, m}^{0}\right)^{1-\theta}\left(\lambda_{j, m}^{1}\right)^{\theta} \\
& =\|\lambda\|_{f_{p(\cdot), q(\cdot)}^{\alpha(\cdot)}\left(\lambda_{j, m}^{0}\right)^{1-\theta}\left(\lambda_{j, m}^{1}\right)^{\theta}}
\end{aligned}
$$

which holds now for all pairs $(j, m)$. As in the proof of Theorem 5, We will prove the following two inequalities

$$
\left\|\lambda^{0}\right\|_{f_{p_{0}(\cdot), q_{0}}^{\alpha_{0}(\cdot)}} \lesssim 1 \quad \text { and } \quad\left\|\lambda^{1}\right\|_{f_{\infty, q_{1}}^{\alpha_{1}(\cdot)}} \lesssim 1
$$

Estimation of $\left\|\lambda^{0}\right\|_{f_{p_{0}(\cdot), q_{0}}^{\alpha_{0}(\cdot)}}$. We write

$$
\sum_{j=0}^{\infty} \sum_{m \in \mathbb{Z}^{n}}\left(2^{j\left(\alpha_{0}(\cdot)+\frac{n}{2}\right)}\left|\lambda_{j, m}^{0}\right| \chi_{j, m}\right)^{q_{0}}=\sum_{\ell=-\infty}^{\infty} \sum_{(j, m) \in C_{\ell}^{\gamma}} \cdots=: I .
$$

We use the local log-Hölder continuity of $\alpha_{0}$ and $\alpha_{1}$ to show that

$$
2^{j u\left(x_{j, m}\right)} \leq c 2^{j u(t)} \quad \text { and } \quad 2^{j \alpha_{0}(x)} \leq c 2^{j \alpha_{0}(t)}, \quad x, t \in Q_{j, m},
$$

where $c>0$ is independent of $\ell$ and $j$. Therefore,

$$
\begin{aligned}
2^{j\left(\alpha_{0}(x)+\frac{n}{2}\right)+j u\left(x_{j, m}\right)} & \lesssim \frac{1}{\left|Q_{j, m} \cap A_{\ell, \gamma}\right|} \int_{Q_{j, m} \cap A_{\ell, \gamma}} 2^{j\left(\alpha_{0}(t)+\frac{n}{2}\right)+j u(t)} d t \\
& \lesssim \frac{1}{\left|Q_{j, m}\right|} \int_{Q_{j, m} \cap A_{\ell, \gamma}} 2^{j\left(\alpha_{0}(t)+\frac{n}{2}\right)+j u(t)} d t \\
& \lesssim \eta_{j, h} * 2^{j\left(\alpha_{0}(\cdot)+\frac{n}{2}\right)+j u(\cdot)} \chi_{Q_{j, m} \cap A_{\ell, \gamma}}(x), \quad j, m \in C_{\ell}^{\gamma}
\end{aligned}
$$


where $h>n$ and the implicit positive constant not depending on $x, \ell, m$ and $j$. Hence $\left\|\left(I^{q_{0}}(\cdot)\right)^{1 / q_{0}}\right\|_{p_{0}(\cdot)}$ is bounded by

$$
c\left\|\left(\sum_{\ell=-\infty}^{\infty} \sum_{(j, m) \in C_{\ell}^{\gamma}}\left(\eta_{j, h} * \digamma_{j, \ell, m}\right)^{q_{0}}\right)^{1 / q_{0}}\right\|_{p_{0}(\cdot)},
$$

where

$$
\digamma_{j, \ell, m}=2^{j\left(\alpha_{0}(\cdot)+\frac{n}{2}\right)+j u(\cdot)+\ell}\left(\frac{\left|\lambda_{j, m}\right|}{\|\lambda\|_{f_{p(\cdot), q(\cdot)}^{\alpha(\cdot)}}}\right)^{\frac{q}{q_{0}}} \chi_{Q_{j, m} \cap A_{\ell, \gamma}} .
$$

Applying Lemma 2 to estimate the last norm by

$$
\begin{aligned}
& c\left\|\left(\sum_{\ell=-\infty}^{\infty} \sum_{(j, m) \in C_{\ell}^{\gamma}} \digamma_{j, \ell, m}^{q_{0}}\right)^{1 / q_{0}}\right\|_{p_{0}(\cdot)} \\
= & c\left\|\left(\sum_{\ell=-\infty}^{\infty} \sum_{(j, m) \in C_{\ell}^{\gamma}} 2^{j\left(\alpha(\cdot)+\frac{n}{2}\right) q+\ell q_{0}}\left(\frac{\left|\lambda_{j, m}\right|}{\|\lambda\|_{f_{p(\cdot), q}^{\alpha(\cdot)}}}\right)^{q} \chi_{Q_{j, m} \cap A_{\ell, \gamma}}\right)^{1 / q_{0}}\right\|_{p_{0}(\cdot)},
\end{aligned}
$$

since $u(\cdot)+\alpha_{0}(\cdot)=\alpha(\cdot) \frac{q}{q_{0}}+\frac{n}{2}\left(\frac{q}{q_{0}}-1\right)$. Observe that

$$
2^{\ell} \leq\left(\frac{g(x)}{\|\lambda\|_{f_{p(\cdot), q}^{\alpha(\cdot)}}}\right)^{\gamma}
$$

for any $x \in Q_{j, m} \cap A_{\ell, \gamma}$ and since $\gamma+\frac{q}{q_{0}}=\frac{p(\cdot)}{p_{0}(\cdot)}$, then $\left\|\left(I^{q_{0}}(\cdot)\right)^{1 / q_{0}}\right\|_{p_{0}(\cdot)}$ is bounded by

$$
\begin{aligned}
& c\left\|\left(\frac{g}{\|\lambda\|_{f_{p(\cdot), q(\cdot)}^{\alpha(\cdot)}}}\right)^{\gamma}\left(\frac{g}{\|\lambda\|_{f_{p(\cdot), q(\cdot)}^{\alpha(\cdot)}}}\right)^{q / q_{0}}\right\|_{p_{0}(\cdot)} \\
= & c\left\|\left(\frac{g}{\|\lambda\|_{f_{p(\cdot), q(\cdot)}^{\alpha(\cdot)}}}\right)^{p(\cdot) / p_{0}(\cdot)}\right\|_{p_{0}(\cdot)} .
\end{aligned}
$$

Obviously, the last norm is less than or equal to one.

Estimation of $\left\|\lambda^{1}\right\|_{f_{\infty, q_{1}}^{\alpha_{1}(\cdot)}}$. By Proposition 1 with $E_{Q_{j, m}}^{\ell}=Q_{j, m} \cap A_{\ell+1, \gamma}^{c}$,

$$
\left\|\lambda^{1}\right\|_{f_{\infty, q_{1}}^{\alpha_{1}(\cdot)}} \lesssim\left\|\left(\sum_{\ell=-\infty}^{\infty} \sum_{(j, m) \in C_{\ell}^{\gamma}} 2^{j\left(\alpha_{1}(\cdot)+\frac{n}{2}\right) q_{1}}\left(\lambda_{j, m}^{1}\right)^{q_{1}} \chi_{E_{Q_{j, m}}^{\ell}}\right)^{1 / q_{1}}\right\|_{\infty} .
$$

Observe that

$$
\lambda_{j, m}^{1} \lesssim 2^{\ell \frac{\delta}{\gamma}+j v(x)}\left(\frac{\left|\lambda_{j, m}\right|}{\|\lambda\| f_{p(\cdot), q}^{\alpha(\cdot)}}\right)^{\frac{q}{q_{1}}} \leq 2^{j v(x)}\left(\frac{g(x)}{\|\lambda\|_{f_{p(\cdot), q}^{\alpha(\cdot)}}}\right)^{-\frac{q}{q_{1}}}\left(\frac{\left|\lambda_{j, m}\right|}{\|\lambda\|_{f_{p(\cdot), q}^{\alpha(\cdot)}}}\right)^{\frac{q}{q_{1}}}
$$

for any $x \in E_{Q_{j, m}}^{\ell}$ and any $(j, m) \in C_{\ell}^{\gamma}$. Therefore, $\left\|\lambda^{1}\right\|_{f_{\infty, q_{1}}^{\alpha_{1}(\cdot)}} \lesssim 1$. Hence, we complete the proof.

Notice that this theorem for $\alpha_{0}=\alpha_{1}=0$ and $q_{0}=q_{1}=2$ was proved by T. Kopaliani, [12, Theorem 3.1]. This theorem can be generalized to the case $0<p_{0}^{+}, p_{1}^{+}, q_{0}, q_{1}<\infty$. 
Suppose that $X_{0}$ and $X_{1}$ are Banach lattices on measure space $(\mathcal{M}, \mu)$, and let $X=$ $X_{0}^{1-\theta} \times X_{1}^{\theta}$ for some $0<\theta<1$. Suppose that $X$ hus the property

$$
f \in X, \quad\left|f_{n}(x)\right| \leq|f(x)|, \mu \text {-a.e., and } \lim _{n \rightarrow \infty} f_{n}=f, \mu \text {-a.e. } \Longrightarrow \lim _{n \rightarrow \infty}\left\|f_{n}\right\|_{X}=\|f\|_{X} \text {. }
$$

Calderón [3, p. 125] then shows that $X_{0}^{1-\theta} \times X_{1}^{\theta}=\left[X_{0}, X_{1}\right]_{\theta}$.

Now we turn to the complex interpolation of the distribution spaces $F_{p(\cdot), p(\cdot)}^{\alpha(\cdot)}$.

Theorem 7 Let $0<\theta<1$. Let $p_{0}, p_{1} \in C^{\log }$ with $1 \leq p_{0}^{+}, p_{1}^{+}<\infty$ and $\alpha_{0}, \alpha_{1} \in C_{\mathrm{loc}}^{\log }$. We put

$$
\frac{1}{p(\cdot)}:=\frac{1-\theta}{p_{0}(\cdot)}+\frac{\theta}{p_{1}(\cdot)} \text { and } \alpha(\cdot):=(1-\theta) \alpha_{0}(\cdot)+\theta \alpha_{1}(\cdot)
$$

Then

$$
\left[f_{p_{0}(\cdot), p_{0}(\cdot)}^{\alpha_{0}(\cdot)}, f_{p_{1}(\cdot), p_{1}(\cdot)}^{\alpha_{1}(\cdot)}\right]_{\theta}=f_{p(\cdot), p(\cdot)}^{\alpha(\cdot)}
$$

and

$$
\left[F_{p_{0}(\cdot), p_{0}(\cdot)}^{\alpha_{0}(\cdot)}, F_{p_{1}(\cdot), p_{1}(\cdot)}^{\alpha_{1}(\cdot)}\right]_{\theta}=F_{p(\cdot), p(\cdot)}^{\alpha(\cdot)}
$$

holds in the sense of equivalent norms.

Proof. Since $f_{p_{0}(\cdot), p_{0}(\cdot)}^{\alpha_{0}(\cdot)}$ and $f_{p_{1}(\cdot), p_{1}(\cdot)}^{\alpha_{1}(\cdot)}$ are Banach lattices. Then, it suffices to use Calderón's result to $X=f_{p(\cdot), p(\cdot)}^{\alpha(\cdot)}$ where the property (21) follows easily from the dominated convergence theorem. Hence Theorem 5 yields (22). Now (23) follows from (22) and Theorem 1 .

Similarly we formulate the main statement on complex interpolation of variable TriebelLizorkin spaces $F_{p(\cdot), q}^{\alpha(\cdot)}$.

Theorem 8 Let $0<\theta<1$ and $1 \leq q_{0}, q_{1}<\infty$. Let $p_{0} \in C^{\log }$ with $1 \leq p_{0}^{+}<\infty$ and $\alpha_{0}, \alpha_{1} \in C_{\mathrm{loc}}^{\mathrm{log}}$. We put

$$
\frac{1}{p(\cdot)}:=\frac{1-\theta}{p_{0}(\cdot)}, \frac{1}{q}:=\frac{1-\theta}{q_{0}}+\frac{\theta}{q_{1}} \text { and } \alpha(\cdot):=(1-\theta) \alpha_{0}(\cdot)+\theta \alpha_{1}(\cdot) .
$$

Then

$$
\left[f_{p_{0}(\cdot), q_{0}}^{\alpha_{0}(\cdot)}, f_{\infty, q_{1}}^{\alpha_{1}(\cdot)}\right]_{\theta}=f_{p(\cdot), q}^{\alpha(\cdot)}
$$

and

$$
\left[F_{p_{0}(\cdot), q_{0}}^{\alpha_{0}(\cdot)}, F_{\infty, q_{1}}^{\alpha_{1}(\cdot)}\right]_{\theta}=F_{p(\cdot), q}^{\alpha(\cdot)}
$$

holds in the sense of equivalent norms.

Using a combination of the arguments used in the proof of Theorems 7 and 8 and the fact that $\frac{\gamma(\cdot)}{\delta(\cdot)}$ is a constant function with negative values, we arrive at the following complex interpolation of variable Triebel-Lizorkin spaces.

Theorem 9 Let $0<\theta<1$. Let $p_{0}, p_{1}, q_{0}, q_{1} \in C^{\log }$ with $1 \leq p_{0}^{+}, q_{0}^{+}, p_{1}^{+}, q_{1}^{+}<\infty$ and $\alpha_{0}, \alpha_{1} \in C_{\mathrm{loc}}^{\log }$. We put

$$
\frac{1}{p(\cdot)}:=\frac{1-\theta}{p_{0}(\cdot)}+\frac{\theta}{p_{1}(\cdot)}, \frac{1}{q(\cdot)}:=\frac{1-\theta}{q_{0}(\cdot)}+\frac{\theta}{q_{1}(\cdot)}, \alpha(\cdot):=(1-\theta) \alpha_{0}(\cdot)+\theta \alpha_{1}(\cdot)
$$


and

$$
\gamma(\cdot):=\frac{p(\cdot)}{p_{0}(\cdot)}-\frac{q(\cdot)}{q_{0}(\cdot)} .
$$

(i) We suppose that $\gamma(x)=0$ for any $x \in \mathbb{R}^{n}$. Then

$$
\left[f_{p_{0}(\cdot), q_{0}(\cdot)}^{\alpha_{0}(\cdot)}, f_{p_{1}(\cdot), q_{1}(\cdot)}^{\alpha_{1}(\cdot)}\right]_{\theta}=f_{p(\cdot), q(\cdot)}^{\alpha(\cdot)}
$$

and

$$
\left[F_{p_{0}(\cdot), q_{0}(\cdot)}^{\alpha_{0}(\cdot)}, F_{p_{1}(\cdot), q_{1}(\cdot)}^{\alpha_{1}(\cdot)}\right]_{\theta}=F_{p(\cdot), q(\cdot)}^{\alpha(\cdot)}
$$

holds in the sense of equivalent norms.

(ii) We suppose that $q_{0}$ and $q_{1}$ are constants. In addition, we assume that $\gamma(x) \neq 0$ for any $x \in \mathbb{R}^{n}$. Then

$$
\left[f_{p_{0}(\cdot), q_{0}}^{\alpha_{0}(\cdot)}, f_{p_{1}(\cdot), q_{1}}^{\alpha_{1}(\cdot)}\right]_{\theta}=f_{p(\cdot), q}^{\alpha(\cdot)}
$$

and

$$
\left[F_{p_{0}(\cdot), q_{0}}^{\alpha_{0}(\cdot)}, F_{p_{1}(\cdot), q_{1}}^{\alpha_{1}(\cdot)}\right]_{\theta}=F_{p(\cdot), q}^{\alpha(\cdot)}
$$

holds in the sense of equivalent norms.

Remark 2 It should be mentioned that if $\alpha_{0}, \alpha_{1}, q_{0}$ and $q_{1}$ are constants, Theorem 4 is more general than what has been given here.

\section{Acknowledgment}

A great deal of this work has been carried out during the visit of the author in Jena, Germany. I wish to thank Professor Winfried Sickel for the hospitality and for many valuable discussions and suggestions about this work.

\section{References}

[1] Almeida, A., Hästö, P.: Interpolation in variable exponent spaces. Rev. Mat. Complut, 27(2), 657-676 (2014)

[2] Bergh, J., Löfström, J.: Interpolation Spaces. An introduction. Springer-Verlag, Berlin, 1976

[3] Calderón, A.P.: Intermediate spaces and interpolation, the complex method. Studia Math, 24, 113-190 (1964)

[4] Cheng, C., Xu, J.: Geometric properties of Banach space valued Bochner-Lebesgue spaces with variable exponent. J. Math. Inequal, 7(3) (2013), 461-475.

[5] Drihem, D.: Some characterizations of variable Besov-type spaces. Annals of Functional Analysis, 6(4), 255-288 (2015)

[6] Drihem, D.: On the duality of variable Triebel-Lizorkin spaces. arXiv:1506.01642

[7] Diening, L., Harjulehto, P., Hästö, P., Mizuta, Y., Shimomura, T.: Maximal functions in variable exponent spaces: limiting cases of the exponent. Ann. Acad. Sci. Fenn. Math, 34(2), 503-522 (2009) 
[8] Diening, L., Hästö, P., Roudenko, S.: Function spaces of variable smoothness and integrability. J. Funct. Anal, 256(6), 1731-1768 (2009)

[9] Diening, L., Harjulehto, P., Hästö, Růžička, M.: Lebesgue and Sobolev spaces with variable exponents. Lecture Notes in Mathematics, vol. 2017, Springer-Verlag, Berlin 2011.

[10] Frazier, M., Jawerth, B.: A discrete transform and decomposition of distribution spaces. J. Funct. Anal, 93(1), 34-170 (1990)

[11] Kalton, N., Mayboroda, S., Mitrea, M.: Interpolation of Hardy-Sobolev-BesovTriebel-Lizorkin spaces and applications to problems in partial differential equations. Contemporary Math, 445, 121-177 (2007)

[12] Kopaliani, T.: Interpolation theorems for variable exponent Lebesgue spaces. J. Funct. Anal, 257, 3541-3551 (2009)

[13] Mendez, O., Mitrea, M.: The Banach envelopes of Besov and Triebel-Lizorkin spaces and applications to partial differential equations. J. Fourier Anal. Appl, 6(5) (2000), 503-531.

[14] Kempka, H., Vybíral, J.: Spaces of variable smoothness and integrability: Characterizations by local means and ball means of differences. J. Fourier Anal. Appl, 18(4), 852-891 (2012)

[15] Noi, T., Sawano, Y.: Complex interpolation of Besov spaces and Triebel-Lizorkin spaces with variable exponents. J. Math. Anal. Appl, 387, 676-690 (2012)

[16] Sickel, W., Skrzypczak, L., Vybíral, J.: Complex interpolation of weighted Besovand Lizorkin-Triebel spaces. Acta. Math. Sci, 30(8), 1297-1323 (2014)

[17] Triebel, H.: Theory of Function Spaces. Birkhäuser Verlag, Basel, 1983

[18] Triebel, H.: Interpolation Theory, Function Spaces, Differential Operators. 2nd edn. (Johann Ambrosius Barth, 1995)

[19] Yang, D., Yuan, W., and Zhuo, C.: Complex interpolation on Besov-type and Triebel-Lizorkin-type spaces. Anal Appl (Singap), (2013), 11: 1350021, 45pp.

[20] Yuan, W., Sickel, W., Yang, D.: Interpolation of Morrey-Campanato and related smoothness spaces. Science China Math, 59(9), 1835-1908 (2015)

[21] Yang, D., Zhuo, C., Yuan, W.: Besov-type spaces with variable smoothness and integrability. J. Funct. Anal, 269(6), 1840-1898 (2015)

[22] Yang, D., Zhuo, C., Yuan, W.: Triebel-Lizorkin type spaces with variable exponents. Banach J. Math. Anal, 9(4), 146-202 (2015)

[23] Xu, J.: Variable Besov and Triebel-Lizorkin spaces. Ann. Acad. Sci. Fenn. Math, 33, 511-522 (2008)

[24] Xu, J.: An atomic decomposition of variable Besov and Triebel-Lizorkin spaces. Armen. J. Math, 2(1), 1-12 (2009) 